Article original

\title{
Identification des facteurs de risques de la macrosomie néonatale à l'EHS «Nouar Fadéla », Oran - Algérie
}

\author{
Identification of the risk factors of neonatal macrosomia in EHS «Nouar Fadéla », \\ Oran - Algeria
}

\author{
Djamila Bouabida', Amina Dida², Fatiha Belaoun², Raouida Mecifi', Feroudja Djeghali' ${ }^{1}$, Nassima Makhlouf \\ ', Feroudja Graine', Malika Benbouabdelah' \\ 'Service de néonatalogie L'EHS « Nouar Fadela »- Oran \\ 2Service Bio statique faculté de Médecine d'Oran \\ 3Service d'épidémiologie L'EHS CANASTEL, Oran
}

Auteur correspondant:med_2230@yahoo.fr

Article reçu le 30/09/2018/Accepté le 30/12/2018

\section{MOTS CLÉS \\ Macrosomie, diabète gestationnel, obésité, souffrance fœtale aigue, mort in utero.}

\begin{abstract}
Résumé
Introduction - Un nouveau-né est dit macrosome si le poids de naissance est supérieur au 90ème percentile selon les courbes de références. C'est une population à risque exposée à des complications néonatales pouvant compromettre le pronostic vital et ou fonctionnel. L'objectif de l'étude était d'estimer la prévalence hospitalière de la macrosomie et identifier les facteurs de risques de survenue de celle-ci.

Patients et méthodes - II s'agit d'une étude transversale, descriptive et analytique, réalisée au niveau du service de néonatalogie " Nouar Fadéla ".
\end{abstract}

Résultats - Durant la période du 15 Avril 2015 au 15 Mars 2017 inclus, 6741 accouchements ont été réalisés. Nous avons colligé 889 nouveau nés ayant fait l'objet de la présente étude dont 425 couples mères-nouveau-nés macrosomes, soit une prévalence hospitalière de 6,3\%. Pour les caractéristiques maternelles : en moyenne, les mères étaient âgées de $30 \pm 5,70$ ans et avaient un $I M C$ de $27,4 \pm 4,9 \mathrm{~kg} / \mathrm{mz}$. La voie haute était l'issue principale de l'accouchement des nouveau-nés macrosomes. Pour les caractéristiques des nouveau-nés : le poids de naissance $(\mathrm{PN})$ a varié entre 4000gr et 5900gr avec un PN moyen de : $4172 \pm 339$ gr. La morbidité néonatale chez les macrosomes est dominée de façon respective par : l'hypoglycémie dans 19,3\% versus $10,6 \%$ chez les eutrophes ; les hématomes profonds dans $3,8 \%$ vs $1,9 \%$ et la paralysie du plexus brachial (PPB) dans $2,1 \%$ versus 0,2 chez les eutrophes. La létalité était notée dans $0,9 \%$ des cas comparée à $0,6 \%$ chez les eutrophes. On a retrouvé un certain nombre d'éléments épidémiologiques intervenant dans la genèse de la macrosomie : l'obésité, le gain pondéral, l'antécédent de macrosomie, le diabète et le terme avancé.

Conclusion - la macrosomie reste une situation à risque, préoccupante du fait de sa morbidité et sa mortalité néonatale. 


\section{KEY WORDS}

Macrosomia, gestational diabetes, obesity, acute fetal distress, death in utero.

\begin{abstract}
Introduction - A newborn is said macrosome if the birth weight is higher than the 90th percentile according to the reference curves. It is a population at risk exposed to neonatal complications that can compromise the vital prognosis and / or functional. The objective of this study was to estimate the hospital prevalence of macrosomia and to identify the risk factors for its occurrence. Patients and methods: this is a cross-sectional, descriptive and analytical study at the «Nouar Fadela» clinic.
\end{abstract}

Results - during the period from April 15, 2015 to March 15, 2017, 6741 deliveries were made. We collected 889 cases that were the subject of the present study including 425 newborn macrosomes, a hospital prevalence of $6.3 \%$. For maternal characteristics: On average, mothers were aged $30 \pm 5.70$ years, and a BMl of $27.45 \pm 4.96$ $\mathrm{kg} / \mathrm{m} 2$. The majority of deliveries of newborns macrosomes was vaginally delivred. For the characteristics of the newborns: the birth weight varied between 4000gr and 5900 gr with an average PNN of: $4172 \pm 339 \mathrm{gr}$. Neonatal morbidity in macrosomes is dominated by: hypoglycemia in $19.3 \%$ versus $10.6 \%$ in eutrophic patients; hematomas in $3.8 \%$ vs $1.9 \%$; PPB in $2.1 \%$ versus 0.2 in eutrophics. Lethality is neonates in $0.9 \%$ of cases compared to $0.6 \%$ in eutrophic A number of epidemiological elements have been found in the genesis of macrosomia: obesity, weight gain, macrosomia history of diabetes mellitus and the advance or late stage.

Conclusion - Macrosomia remains a risky situation, which is worrying because of its morbidity and neonatal mortality.

\section{Introduction}

L La macrosomie fœtale (MF) est habituellement définie par un poids de naissance supérieur à 4000 grammes, grâce aux classiques courbes de poids en fonction du terme, in utero par échographie ou après naissance) [1]. Ainsi, un nouveau-né (NN) est macrosome lorsque son poids de naissance est supérieur au 90e percentile pour l'âge gestationnel (AG) selon les courbes de référence pour une population donnée qui correspond au terme anglo-saxon Large for Gestationnel Age (LGA, poids élevé pour l'âge)[1].

La macrosomie est hétérogène en termes de phénotype, clinique et biologique, et en termes d'étiologies.

La macrosomie constitue un réel problème de santé publique lié non seulement à sa prévalence dans les pays développés et ceux en développement, mais aussi à sa forte association avec la morbi-mortalité infantile (complications traumatiques, hématologiques, métaboliques...).

À travers ce travail notre objectif était d'estimer la fréquence de la macrosomie néonatale le service de néonatalogie à l'EHS Nouar Fadéla et d'identifier les facteurs de risque de survenue de celle-ci: âge maternel, antécédent d'un accouchement d'un macrosome, obésité, diabète...).

\section{Patients et méthodes}

Il s'agit d'une étude transversale, nichée dans la cohorte des femmes enceintes ayant accouché durant la période du 15 Avril 2015 au 15 Mars 2017 inclus au niveau du service de néonatalogie Nouar Fadéla.

Tous les nouveaux-nés à terme (supérieur à 37 semaines d'aménorrhée (SA), dont le poids de naissance était supérieur au 50e percentile pour l'âge gestationnel sont recrutés dans l'étude. Les mort-nés macrosomes (difficulté de déterminer leur âge gestationnel) et les nouveau-nés de moins de 37 SA ont été exclus de l'échantillon.

Nous avons mis au point des fiches d'enquête qui ont été remplies à travers un interrogatoire auprès de la maman. Elles comprennent trois parties :

- les caractéristiques maternelles : âge, profession, situation matrimoniale, niveau socio-économique, parité, gestité, antécédents obstétricaux (avortement, mort-né, diabète antérieur, diabète grossesse),

- les caractéristiques de la grossesse actuelle et l'accouchement (poids au début et fin de grossesse (poids prégestationel, gain pondéral), taille maternelle, tension artérielle, dépistage de diabète (hypoglycémie, hémoglobine glyquée, 
glycémie veineuse), suivi de grossesse, antécédent de macrosomie),

- et les caractéristiques des nouveau-nés (poids, taille, périmètre crânien) et les complications néonatales actuelles.

\section{Analyse statistique}

La saisie des données ainsi que l'analyse descriptive et analytique des données ont été effectuées à l'aide de logiciel Statistical Package for Social Sciences (SPSS) version 20. Le test de Chi 2 a été utilisé pour la comparaison des résultats. Le seuil de $p<0,05$ était considéré comme statistiquement significatif.

\section{Résultats}

Nous avons colligé 889 cas ayant fait l'objet de la présente étude dont 425 couples mères-nouveau-nés macrosomes soit une prévalence hospitalière de $6,3 \%$.

Caractéristiques maternelles : L'âge maternel a varié entre 26 ans et 45 ans avec une moyenne d'âge 30,33 \pm 6,03 ans. Par contre, la tranche d'âge prédominante était entre 26 et 35 ans.

En ce qui concerne la taille maternelle, elle a varié entre $145 \mathrm{~cm}$ et $175 \mathrm{~cm}$ avec une moyenne dans notre échantillon de $163,9 \pm 6,4 \mathrm{~cm}$. La tranche de taille prédominante était plus $165 \mathrm{~cm}$ avec un taux de 80,7\% chez les mères des nouveau-nés macrosomes vs $76 \%$ chez les mères des nouveau-nés eutrophiques

La multiparité était observée dans $44,2 \%$ des mères des nouveau nés macrosomes vs $55,8 \%$ des nouveau nés des mères eutrophes. Dans notre population d'étude, l'obésité représente $24,9 \%$ chez les mères des nouveau-nés macrosomes vs $14,4 \%$ chez les mères des NN eutrophes.

Toutefois, nous considérons $15 \mathrm{~kg}$ comme prise de poids limite au cours de la grossesse, nous avons trouvé 75 (17,6 $\%$ des mères des nouveau-nés macrosomes qui ont eu une prise de poids excessive. Les parturientes des mères des nouveau-nés macrosomes à terme (AT) représentaient $291(68,5 \%)$ cas, alors que $134(31,5 \%)$ cas avaient un dépassement du terme (DT).

\section{Antécédents maternels médicaux}

L'antécédent de macrosomie (40\% versus $11,4 \%):(p<$ 0,0001 ) est l'antécédent le plus caractéristique dans notre population étudiée avec une différence significative $(P<$
$0,0001)$ suivi de diabète gestationnel $(20,7 \%$ vs $10,3 \%): p$ $<0,0001$ et diabète chronique $(5,4 \%$ vs $2,2 \%)(p=0.01)$ (Tableau 1).

Tableau 1. Antécédents maternels médicaux

\begin{tabular}{|c|c|c|c|c|c|}
\hline \multirow{2}{*}{ ATCD } & \multicolumn{2}{|c|}{$\begin{array}{c}\text { Macrosomes } \\
\mathrm{N}=425\end{array}$} & \multicolumn{2}{c|}{ Non Macrosomes } & \multicolumn{2}{c|}{$\begin{array}{c}\text { Seuil de } \\
\text { signification } \\
\text { P }\end{array}$} \\
\cline { 2 - 5 } & $\mathrm{N}$ & $\%$ & $\mathrm{~N}$ & $\%$ & $\mathrm{~N}$ \\
\hline $\begin{array}{c}\text { Avortement } \\
\text { (ABRT) }\end{array}$ & 69 & 16,2 & 60 & 12,9 & $\mathrm{NS}$ \\
\hline $\begin{array}{c}\text { Mort in utero } \\
\text { (MIU) }\end{array}$ & 19 & 4,5 & 22 & 4,7 & $\mathbf{2}$ \\
\hline $\begin{array}{c}\text { Diabète } \\
\text { gestationnel }\end{array}$ & 88 & 20,7 & 48 & 10,3 & $<0,0001$ \\
\hline $\begin{array}{c}\text { Diabète } \\
\text { chronique }\end{array}$ & 23 & 5,4 & 10 & 2,2 & $<0,01$ \\
\hline $\begin{array}{c}\text { Hypertension } \\
\text { artérielle(HTA) }\end{array}$ & 79 & 18,6 & 72 & 15,5 & $\mathrm{NS}$ \\
\hline $\begin{array}{c}\text { Antécédent } \\
\text { Macrosomie }\end{array}$ & 170 & 40,0 & 53 & 11,4 & $<0,0001$ \\
\hline
\end{tabular}

Evolution du travail et décision du mode d'accouchement

La majorité des accouchements des nouveau nés macrosomes a été par voie haute soit 314 cas $(73,3 \%)$ vs 111 cas $(26,1 \%)$ par voie basse avec une différence significative $(p=0,005)$. La césarienne a été indiquée respectivement dans les cas représentés de la Figure 1.

Figure 1. Répartition des mères selon l'indication de la césarienne

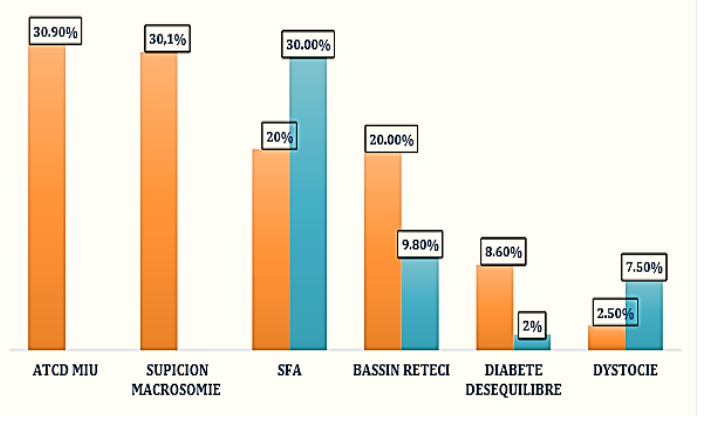

Caractéristiques des nouveau-nés

On note une prédominance masculine qui était de $61,7 \%$ et $58,8 \%$ respectivement chez les macrosomes et les eutrophes. Le poids de naissance (PNN) a varié entre 4000gr et 5900gr avec un PNN moyen de : $4172 \pm 339$ gr vs le poids de naissance des témoins a varié entre $2380 \mathrm{gr}$ et $3800 \mathrm{gr}$ avec un PNN moyen de : $3296 \pm 278$ gr $(p<0,0001)$. (Figure .2) 
Figure2. Les paramètres des nouveau-nés macrosomes

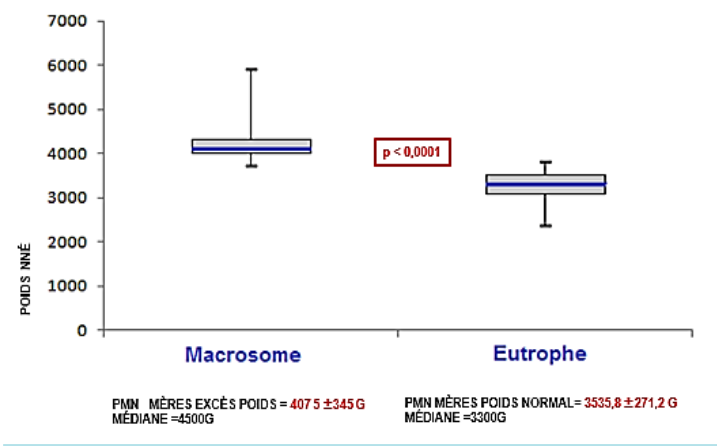

Répartition des morbidités néonatale

En ce qui concerne les complications néonatales : $66 \%$ ont été remis à leur maman et $34 \%$ nouveau-nés ont été hospitalisés.

La morbidité néonatale chez les macrosomes par rapport aux eutrophes par ordre croissant est dominée par :

- L'hypoglycémie : 82 cas $(19,3 \%)$ versus $49(10,6 \%)$ avec $P<0,0001$ et $\mathrm{OR}=2,025(1,38-2,96)$

- Fracture humérale : 5 cas (1,2\%) versus 0 cas les non macrosomes avec $p=0,01$ PPB : 9 cas $(2,1 \%)$ vs 1 cas $(0,2 \%)$ avec $p<0,007$ et $\mathrm{OR}=10,01 \quad(1,26-79,39) \quad$ (Tableau 2)

Tableau 2. Répartition des morbidités néonatales

\begin{tabular}{|c|c|c|c|c|}
\hline \multirow{2}{*}{ Complications néonatales } & \multicolumn{2}{|c|}{ Macrosomes } & \multirow{2}{*}{$\begin{array}{c}\text { Seuil de } \\
\text { signification } \\
\text { P }\end{array}$} & \multirow[t]{2}{*}{ Odds/ ratio } \\
\hline & $\begin{array}{l}\text { Oui } \\
\mathrm{N}(\%)\end{array}$ & $\begin{array}{l}\text { Non } \\
\mathrm{N}(\%)\end{array}$ & & \\
\hline Hypoglycémie $(<0,40 \mathrm{gr} / \mathrm{l})$ & $82(19,3)$ & $49(10,6)$ & $<0,0001$ & $\begin{array}{l}2,025[1,38- \\
2,96]\end{array}$ \\
\hline Hypocalcémie $(<80 \mathrm{mg} / \mathrm{l})$ & $7(1,6)$ & $16(3,4)$ & NS & NS \\
\hline Hyperbilirubinémie & $45(10,6)$ & $115(24,8)$ & $<0,0001$ & $\begin{array}{l}1,85[1,03- \\
2,03]\end{array}$ \\
\hline Polyglobulie & $1(0,2)$ & $2(0,4)$ & NS & NS \\
\hline Asphyxie périnatale (APN) & $23(5,4)$ & $45(9,7)$ & NS & NS \\
\hline Détresse respiratoire & $42(9,9)$ & $124(26,7)$ & $<0,0001$ & $\begin{array}{c}2,09[1,59- \\
2,74]\end{array}$ \\
\hline $\begin{array}{c}\text { Cardiomyopathie } \\
\text { hypertrophique (CMH) }\end{array}$ & $3(0,7)$ & $4(0,9)$ & NS & NS \\
\hline $\begin{array}{l}\text { Paralysie du plexus } \\
\text { brachial } \\
\text { (PPB) }\end{array}$ & $9(2,1)$ & $1(0,2)$ & 0,007 & $\begin{array}{c}10,01[1,26- \\
79,39]\end{array}$ \\
\hline Fracture clavicule & $3(0,7)$ & $1(0,2)$ & NS & \\
\hline Fracture humérale & $5(1,2)$ & $0(0)$ & & \\
\hline Bosse séro sanguine & $37(8,7)$ & $58(12,5)$ & NS & \\
\hline Hématomes & $16(3,8)$ & $9(1,9)$ & NS & \\
\hline Décès & $5(0,9)$ & $3(0,6)$ & NS & \\
\hline
\end{tabular}

Les facteurs de risques de la macrosomie néonatale

- L'obésité était de 55,5\% chez les mères des macrosomes versus $45,5 \%$ mères des eutrophes avec une différence significative $: P<0,0001$ avec $O R=2,53(1,74-3,66)$.

- En considérant $15 \mathrm{~kg}$ comme prise de poids limite au cours de la grossesse, on a trouvé 75 patientes qui ont eu une prise de poids excessive avec un poids antérieur bien connu soit un taux de $17,6 \%$ vs $10,8 \%$ du groupe témoin $(p=0,004$, OR= $1,77[1,2-2,61])$.

- En ce qui concerne les antécédents médicaux et obstétricaux, comparativement aux mères de témoins, les mères de macrosomes ont plus significativement un accouchement antérieur d'un macrosome ( $40 \%$ vs $11,4 \%$ témoins) $(p<0,0001)$ et le risque est multipliée par OR : 5,17 [3,65 - 7,30] .

- Le sexe masculin $(72,9 \%)$ est plus fréquent que le sexe féminin $(27,1 \%)$ cette différence est statistiquement significative avec $O R=1,88[1,42-2,5](p<0,0001)$ par rapport aux témoins.

- Le diabète est respectivement de $20,7 \%$ chez les mères macrosomes ( $M M)$ comparé à $10,3 \%$ chez les mères témoins(MT) la différence est statistiquement significative avec un $(p<0,0001)$ et le risque est multiplié par $O R=2,26$ [1,54- 3,31].

- Terme avancé était important chez le groupe de mères macrosomes vs témoins $O R=1,84[1,32-2,57]$ suivi par gain pondéral $O R=1,65[1,22-2,23]$.

- Notre étude montre que les proportions des accouchées âgées de 35 ans ou plus étaient de 23,5\% chez les mères de macrosomes contre $20,5 \%$ chez les mères des nouveau nés eutrophes. La comparaison de ces proportions donne une différence statistiquement non significative. ( $P=N S)$ signifiant que l'Age est non significativement associé à l'accouchement d'un macrosome.

- Il ressort aussi que la proportions de multipares était chez les mères des eutrophes que chez les mères des macrosomes $(57,1 \%$ et $49,4 \%)$,la différence est significative OR $: 1,75[1,33-2,32]$ et $P=0,0001$.

La taille n'a pas d'influence sur le poids du nouveau par contre obésité paternelle influence le poids du nouveau-né avec une différence significative $(P=0,003)$. (Tableau 3) 
Tableau 3. Analyse multi variée des facteurs de risques de la macrosomie retenus

\begin{tabular}{|c|c|c|c|c|c|c|c|}
\hline \multirow{2}{*}{\multicolumn{2}{|c|}{$\begin{array}{l}\text { Facteurs de risque de } \\
\text { macrosomie }\end{array}$}} & \multicolumn{2}{|c|}{$\begin{array}{c}\text { Macrosomes } \\
N=425\end{array}$} & \multicolumn{2}{|c|}{$\begin{array}{l}\text { Non Macrosomes } \\
\qquad N=464\end{array}$} & \multicolumn{2}{|c|}{ ANALYSE MULTI VARIÉE } \\
\hline & & $\mathrm{N}$ & $(\%)$ & $N$ & $\%$ & \multirow{2}{*}{$\begin{array}{c}\text { OR BRUT [C a a 95\%] } \\
1\end{array}$} & \multirow[t]{2}{*}{ P.Value } \\
\hline Sexe de & Masculin & 310 & $(72,9)$ & 273 & $(58,8)$ & & \\
\hline l'enfant & Féminin & 115 & $(27,1)$ & 191 & $(41,2)$ & $2,168[1,501 \cdot 2,802]$ & $<0,0001$ \\
\hline \multirow[t]{5}{*}{ IMC de la mère } & Dénutrition & 02 & 0,5 & 9 & 1,9 & $0,477[0,099 \cdot 2,293]$ & NS \\
\hline & Normal & 145 & 34,1 & 232 & 50 & 1 & \\
\hline & Surpoids & 172 & 40,5 & 156 & 33,6 & $1,575[1,138-2,178]$ & \\
\hline & Obésité & 106 & 24,9 & 67 & 14,4 & $1,816[1,198 \cdot 2,752]$ & 0,005 \\
\hline & $37 \cdot 395 \mathrm{~A}$ & 291 & $(68,5)$ & 363 & $(78,2)$ & 1 & \\
\hline \multirow[t]{2}{*}{ Terme } & $40.425 \mathrm{~A}$ & 134 & $(31,5)$ & 101 & $(21,8)$ & $1,81[1,30-3,11]$ & $<0,0001$ \\
\hline & Non & 337 & $(79,3)$ & 416 & $(89,7)$ & 1 & \\
\hline Diabète & Oui & 88 & $(26,1)$ & 48 & $(10,3)$ & $2,070[1,37-2,96]$ & $<0,0001$ \\
\hline \multirow[t]{2}{*}{ Gain pondéral } & $<15 \mathrm{KG}$ & 350 & $(82,4)$ & 414 & $(89,2)$ & 1 & \\
\hline & $>15 \mathrm{KG}$ & 75 & $(17,6)$ & 50 & $(10,8)$ & $1,80[1,18-2,75]$ & 0,006 \\
\hline ATCD de & Non & 255 & $(60,0)$ & 411 & $(88,6)$ & 1 & $<0,0001$ \\
\hline Macrosomie & Oui & 170 & $(40,0)$ & 53 & $(11,4)$ & $4,41[3,06-6,36]$ & \\
\hline
\end{tabular}

\section{Discussion}

Dans notre étude la prévalence hospitalière de la macrosomie néonatale était de $6,3 \%$. Nos résultats restent proches de ceux retrouvés par la majorité des études [1]. Par contre, certains auteurs rapportent une fréquence plus élevée [1,2]. Cette augmentation peut être liée à un gain de poids maternel plus élevé pendant la grossesse, à l'augmentation des fréquences de l'obésité maternelle et du diabète, et le régime alimentaire. Mais d'autres auteurs rapportent une fréquence plus faible notamment dans les pays d'Afrique [3].Ces variations de fréquence entre les séries pourraient s'expliquer par la taille de l'échantillon, le suivi insuffisant, le manque d'hygiène de vie au cours de la grossesse ainsi que le bas niveau socio-économique [3-4].

Comparativement aux données de littérature, l'âge et la multiparité n'ont pas été statistiquement significatifs comme facteurs de risque dans notre étude.

Le dépassement du terme était observé chez 32\% des mères d'enfants macrosomes ce qui rejoint la plupart des études [5]. Cela peut être expliqué par un défaut d'une échographie précoce en vue d'une datation de la grossesse, l'imprécision de dates des dernières règles et le caractère irrégulier des consultations prénatales [1,5-6].

Quant au taux de $25 \%$ de cas d'obésité retrouvés dans notre série, il rejoint celui constaté dans la littérature [7]. Et le risque d'accoucher un macrosome est multiplié 2 chez les gestantes en surpoids et les obèses. Alors que dans la littérature ce risque est multiplié par 3 avec un intervalle de prise pondérale variant entre $12-18 \mathrm{Kg}$ [8]. Dans notre série la prise pondérale était de $15 \mathrm{Kg}$ et le risque est multiplié par 2. La prise de poids excessive peut être expliquée par une modification du métabolisme maternel car dépendante de l'alimentation expliquant ainsi la macrosomie par anabolisme $[9,10]$.

Quel que soit le type de diabète, nos résultats rejoignent ceux de la littérature [9], mais le pourcentage de diabète gestationnel dans notre série est plus élevé par rapport aux études. Cela peut être expliqué surtout par les variations ethniques et génétiques des populations, mais également, dans une moindre mesure, par la diversité des stratégies et des méthodes de dépistage utilisées $[10,11]$.

L'antécédent de macrosomie est le facteur le plus incriminé. Cependant, sa physiopathologie n'est pas encore élucidée. Ceci nous confirme qu'une femme ayant accouché d'un macrosome récidive le plus souvent avec un risque multiplié par six $(O R=6)$ et dans notre série, celui ci est multiplié par $5[4,11-13]$.

La prédominance masculine a été rapportée par la plupart des auteurs, avec un risque relatif multiplié par deux et nos résultats rejoignent la plupart des études [11]. Selon cellesci, le fœtus féminin est génétiquement plus résistant à l'insuline et moins sensible aux effets trophiques de l'insuline et est donc plus petit $[11,17,19]$ (Tableau 3).

La majorité d'accouchements d'enfants macrosomes a été eutocique (73,9\% vs $65,7 \%$ témoins) par voie haute avec une différence significative. Ce taux de césarienne dans le groupe de macrosomes est élevé par rapport à celui trouvé par la plupart des études [20]. Par conséquent, la macrosomie multiplie le risque de césarienne $(\mathrm{p}=0,005)$. Ce taux varie selon les études $[20,22]$.

Comme en témoigne la majorité des séries marocaines, l'accouchement d'un nouveau-né macrosome est d'abord spontané. Le recours au forceps est rare, contrairement aux pays Européens et Américains, où l'utilisation du forceps est plus fréquente [20,23-24].

Concernant la morbidité néonatale, celle-ci est dominée par l'hypoglycémie et nos résultats rejoignent ceux de la littérature [25].

Il existe une relation linéaire entre le poids de naissance, la dystocie des épaules et le PPB ceci concorde avec nos résultats [26-27].

La létalité est notée dans $1 \%$ des cas dans notre série. Le 
taux global de mortalité néonatale, dans notre étude, rejoint celui trouvé par certaines études. Ceci s'expliquerait du fait que la plupart des grossesses n'étaient pas suivies [28-29].

Cette étude a des limites. Premièrement, les données ont été recueillies rétrospectivement à partir d'un seul centre plutôt que de plusieurs centres. Par conséquent, notre échantillon ne pouvait pas être représentatif et les résultats ne peuvent pas être généralisés.

Aussi, la comparaison de nos résultats avec d'autres études devrait être faite avec prudence. En fait, les différences peuvent être liées à des différences de méthodologie et/ou de taille de l'échantillon et de définition de la macrosomie du point de vue pédiatrique.

\section{Conclusion}

La macrosomie reste une situation à risque, préoccupante du fait de sa morbidité et sa mortalité néonatale. Plusieurs facteurs maternels peuvent influencer sa survenue .Dans notre contexte On a retrouvé un certain nombre d'éléments épidémiologiques intervenant dans la genèse de la macrosomie : l'obésité, le gain pondéral, l'antécédent de macrosomie, le diabète et le terme avancé.

afin d'améliorer le pronostic fœto-maternel. Il faut premièrement, :Motiver le suivi régulier de toutes les grossesses. Deuxièmement, Le dépistage du diabète gestationnel afin de. Bien traiter et équilibrer le diabète pour les femmes diabétiques. Troisièmement, Surveiller le gain pondéral gravidique et l'améliorer à l'aide de nouveaux outils simples d'utilisation, à l'instar des courbes de poids maternel et une collaboration entre les professionnels de la nutrition, la diététicienne et le nutritionniste, est à approfondir, afin d'optimiser la prise en charge des femmes.

À partir de cette étude nos perspectives sont de :

Dépister les gestantes à risque d'accoucher un macrosome afin d'améliorer la qualité de vie, de diminuer les dépenses de santé, évaluer les performances du dépistage de la macrosomie fœtale et mesurer l'impact sur les pratiques obstétricales ,prise en charge multidisciplinaire de l'accouchement et établir un protocole de prise en charge des macrosomes avec complications.

\section{Conflits d'intérêt}

Les auteurs déclarent n'avoir aucun conflit d'intérêt.

\section{Références bibliographiques}

[1]. FOETALE. J. GYNECOLOBSTETBIOLREPROD 2000 : 29 (SUPPL N¹) : 6-12

[2]. GROSSETTI E, BEUCHER G, REGEASSEA,LAMENDOUR N, DREYFS M. COMPLICATIONS OBSTETRICALES DE L'OBESITE MORBIDE. J GYNECOLOBSTETBIOLREPROD 2004; 33: 739.744

[3]. SURVEY.AIKOYANAGI, JUN ZHANG, AMARJARGALDAGVADORJ, FUMI HIRAYAMA, KENJI SHIBUYA, JOÃO PAULO SOUZA, AHMETMETINGÜLMEZOGLU LANCET, MACROSOMIA IN 23 DEVELOPING COUNTRIES: AN ANALYSIS OF A MULTICOUNTRY, FACILITY-BASED, CROSS-SECTIONAL 2013; 381: $476-83$

[4]. FATNASSI R. ET ALL ,PAN AFRICAN MEDICAL JOURNAL. 2017;28:126. DOl:10.11604/ PAMJ.2017.28.126.8508

[5. PANEL P, DE MEUS JB, YANOLOPOULOSB,MAGNIN G. ACCOUCHEMENT DU GROS ENFANT. J.GYNECOL. OBSTET. BIOL. REPROD $1991 ; 20: 729-736$.

[6]. MATTHEW C, NELL J, JOHN P. RISK FACTORS FOR MACROSOMIA A DITZ CLINICAL CONSEQUENCE: A STUDY OF 350,311 PREGNANCIES. EUR J OBSTETGYNECOLREPRODBIOL 2003; 111; 1:9-14

[7]. COULLBALY ETIENNE Y. ACCOUCHEMENT DU GROS FOETUS AU CHU GABRIELTOURE: FACTEURS DE RISQUE ET PRONOSTIC MATERNO-FOETAL. [MALI];THESE DE MEDECINE 2008-2009 .

[8]. BALLARD JL, ROSENN B, KHOURYJC, MIODOVNIK M. DIABETIC FETAL MACROSOMIA:SIGNIFICANCE OF DISPROPORTIONATE GROWTH. J PEDIATR. 1993;122(1):115-119.

[9]. WORLD HEALTH ORGANIZATION (WHO): RECOMMENDED DEFINITION TERMINOLOGY AND FORMAT FOR STATISTICAL TABLES RELATED TO THE PERINATAL PERIOD AND RISE OF ANEW CERTIFICATION FOR CAUSE OF PERINATAL DEATHS. MODFIICATIONS RECOMMENDED BY FIGO AS AMENDED, OCTOBER 14, 1976. ACTAOBSTETGYNECOLSCAND 56:347, 1977.

[10]. BEN-HAROUSH A., YOGEV Y., HOD M. EPIDEMIOLOGYOF GESTATIONAL DIABETES MELLITUS AND ITS ASSOCIATION WITH TYPE 2 DIABETES DIABET. MED. $2004 ; 21$ : $103-113$

[11]. MIMOUNI .N ,PROFLL EPIDEMIOLOGIQUE DES NOUVEAUX NES DES MERESDIABETIQUES AU SERVICE DE NEONATOLOGIE DE L'EHSMERE-ENFANT DE TLEMCEN BERRANDOU, THESE DE MEDECINE 2015

[12]. WILKINTJ, MURPHY MJ. L'HYPOTHESE DE L'INSULINE DE GENRE: POURQUOI LES FILLES NAISSENT-ELLES PLUS LEGERES OUE LES GARCONS, ET L'IMPLICATION DE LARESISTANCEAL'INSULINE. INT J OBES. 2006; 30: 1056-61.

[13]. DOLO A. ACCOUCHEMENT DU GROS FOETUS AU SERVICE DE GYNECOLOGIEOBSTETRIQUE DU CENTRE HOSPITALER UNIVERSITAIRE DU POINT G. A PROPOS DE 205 CAS THES.MED.: BAMAKO, 2001, 69P. ; N 85

[14]. MAGNIN G. L'ACCOUCHEMENT DU GROS ENFANT. PRAT ACCOUCHEMENT I LANSAC G BODYEDSVILERBANNESIMEPEDN. 1989;149.

[15]. ORSKOU J., ET AL., MATERNAL CHARACTERISTICS AND LIFESTYLE FACTORS AND THE RISK OF DELIVERING HIGH BIRTH WEIGHT INFANTS. OBSTETRICS AND GYNECOLOGY, 2003. 102(1): P. 115-20.

[16]. CUIFANG .F ET ALLPATERNAL FACTORS TO THE OFFSPRING BIRTH WEIGHT: THE 829BIRTH COHORT STUDY.INT J CLINEXP MED 2015;8(7):11370-11378

[17]. GERARD G. NAHUM, M.D., AND HAROLD STANISLAW, PH.D.RELATIONSHIP OF PATERNAL FACTORS TO BIRTH WEIGHT FROM THE DEPARTMENT OF OBSTETRICS AND GYNECOLOGY., THE JOURNAL OF REPRODUCTIVE MEDICINE®. VOLUME 48, NUMBER 12/DECEMBER 2003

[18]. FETTAHMERREM , LA MACROSOMIE FOETALE A TERME (A PROPOS DE 340 CAS) THESEPRESENTEE ET SOUTENUE PUBLLQUEMENT LE 29/06/2016 FES, MAROC

[19]. ANDERSEN J., ET AL., PERINATAL BRACHIAL PLEXUS PALSY. PAEDIATRICS\& CHILD HEALTH, 2006.11(2): P. 93-100.

[20]. AISHA SALIM SAID , RISK FACTORS AND OUTCOMES OF FETAL MACROSOMIA IN A TERTIARY CENTRE IN TANZANIA:A CASE-CONTROL STUDY, SAID AND MANJIBMC PREGNANCY AND CHILDBIRTH (2016) 16:243

[21]. CISSESANOGO A. ETUDE EPIDEMIO-CLINIQUE DE LA MACROSOMIE FOETALE AU CENTRE DE SANTE DE REFERENCE DE LA COMMUNE II THESE MED. BAMAKO, 2009.

[22]. BATTAGLIA FC, MESCHIA G. FETAL NUTRITION. ANNUREVNUTR 1988;8:43-61.

[23]. KABALI C. AND WERLER M.M., PRE-PREGNANT BODY MASS INDEX, WEIGHT GAIN AND THE RISKOF DELIVERING LARGE BABIES AMONG NON-DIABETIC MOTHERS. INTERNATIONAL JOURNAL OF GYNAECOLOGY AND OBSTETRICS: THE OFFICIAL ORGAN OF THE INTERNATIONAL FEDERATION OF GYNAECOLOGY AND OBSTETRICS, 2007. 97(2): P. 100-4.

[24]. FREDERICK I.O., ET AL., PRE-PREGNANCY BODY MASS INDEX, GESTATIONAL WEIGHT GAIN, AND OTHER MATERNAL CHARACTERISTICS IN RELATION TO INFANT BIRTH WEIGHT. MATERNALAND CHILD HEALTH JOURNAL, 2008. 12(5): P. 557-67.

[25]. PHELAN JP, EGLINTONGS, HORENSTEINJM, CLARK SL, YEH S. PREVIOUS CESAREAN BIRTH TRIAL OF LABOR IN WOMEN WITH MACROSOMIC INFANTS. J REPROD MED. 1984 JAN;29(1):36-40. [26]. MAGNIN G, OURYJF, SIBONY O. ACCOUCHEMENT DU GROS ENFANT. IN: LANSAC J, DESCHAMPS P, OURYJF, EDITORS. PRATIQUE DE L'ACCOUCHEMENT. 5E EDITION. PARIS: ELSEVIER MASSON; 2011. PP. 227-236.

[27]. FETTAHM.LAMACROSOMIE FOETALE A TERME(A PROPOS DE 340 CAS) THESE 2016

[28]. EL BARNOUSSI L, FAROUQI A, SAADI N, CHRABI C, BEZAD R, ALAOUI MT. L'ACCOUCHEMENT DU MACROSOME: A PROPOS DE 353 CAS. MEDECINE DU MAGHREB. 2008;158:65-69.

[29]. BATALLAN A, GOFFINET F, PARIS-LLADO J, FORTIN A, BREART G, MADELENAT P, BENIFLAJL, LE GROUPE D'ETUDE DES MATERNITES PARIIIENNES MACROSOMIE FCETALE: PRATIQUES,

[30]. CONSEQUENCES OBSTETRICALES ET NEONATALES - ENQUETE MULTICENTRIQUE CAS-TEMOINS MENEE DANS 15 MATERNITES DE PARIS ET D'ILE DE FRANCE. GYNECOLOGIE OBSTETRIQUE \& FERTILITE 2002;30(6):483-491. [PUBMED]. 